Using interior point solvers for optimizing progressive lens models with spherical coordinates

Glòria Casanellas

Jordi Castro

Horizons Optical SLU

Dept. of Stat. and Oper. Res.

Sant Cugat del Vallès, Barcelona

Universitat Politècnica de Catalunya

Barcelona, Catalonia

gloria.casanellas@horizonsoptical.com

jordi.castro@upc.edu

Research Report UPC-DEIO DR 2019-01

May 2019

Report available from http://www-eio.upc.es/ jcastro 



\title{
Using interior point solvers for optimizing progressive lens models with spherical coordinates
}

\author{
Glòria Casanellas · Jordi Castro
}

\begin{abstract}
Designing progressive lenses is a complex problem that has been previously solved by formulating an optimization model based on Cartesian coordinates. In this work a new progressive lens model using spherical coordinates is presented, and interior point solvers are used to solve this new optimization model. Although this results in a highly nonlinear, nonconvex, continuous optimization problem, the new spherical coordinates model exhibits better convexity properties compared to previous ones based on Cartesian coordinates. The real-world instances considered gave rise to nonlinear optimization problems of about 900 variables and 15000 constraints. Each constraint corresponds to a point of the grid used to define the lens surface. The number of variables depends on the precision of a B-spline basis used for the representation of the surface, and the number of constraints depends on the shape and quality of the design. We present results of progressive lenses obtained using the AMPL modeling language and the nonlinear interior point solvers IPOPT, LOQO and KNITRO. Computational results are reported, as well as some examples of real-world progressive lenses calculated using this new model. Progressive lenses obtained are competitive in terms of quality with those resulting from previous models that are used in commercial glasses.
\end{abstract}

Keywords Nonlinear Optimization, Interior Point Methods, Optical Lens Design, Progressive Lenses, Optimization Industry Applications.

Glòria Casanellas

Horizons Optical SLU, Av. Alcalde Barnils 72, 08174 Sant Cugat del Vallès, Spain. E-mail: gloria.casanellas@horizonsoptical.com

Jordi Castro*

Dept. of Statistics and Operations Research, Universitat Politècnica de Catalunya, Edifici C5, Planta 2, Campus Nord, C. Jordi Girona 1-3, 08034 Barcelona, Catalonia. E-mail: jordi.castro@upc.edu. 

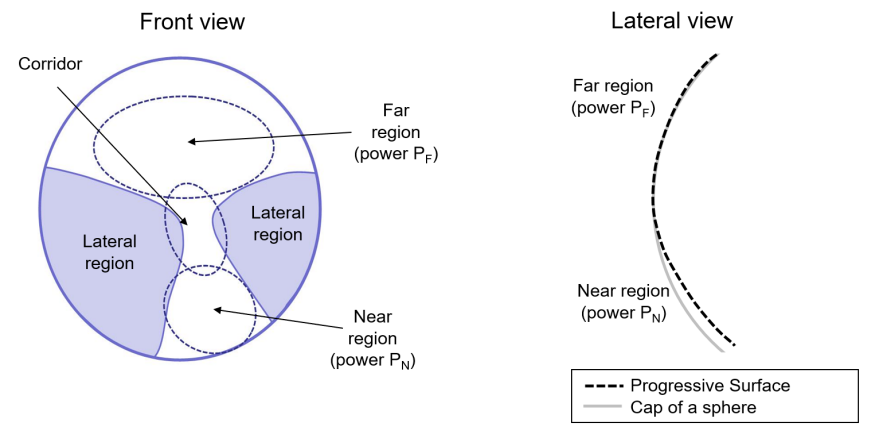

Fig. 1 Parts of a progressive lens.

\section{Introduction}

Presbyopia is the gradual inability of the eyes to focus on near objects. It appears around forties and lenses are required in order to see correctly in near vision. Progressive lenses correct presbyopia and have a complex design: have an upper region for far vision (far region), the corridor for middle vision and the low region for near vision (near region). The different parts of a progressive lens surface are showed in Figure 1 (left). The two main properties of a progressive lens are the power and the astigmatism (whose formulae will be provided in the next Section), defined at each point of the lens. In geometrical terms, the optical power is the mean of the principal curvatures of the surface lens multiplied by a constant, and the astigmatism is the difference of the principal curvatures multiplied by the same constant. When the power changes vertically, unwanted lateral astigmatism (aberrations) appears due to Minkwitz theorem [10. The near zone has to have a bigger power $\left(P_{N}\right)$ than the far region $\left(P_{F}\right)$, and the corridor has a gradual increase of power. Optimization techniques are used to design this type of surfaces, minimizing lateral aberrations.

In order to have stable far and near regions, their curvatures must be almost constant. Consequently, far and near regions should be similar to the caps of two spheres, the radius of the sphere of the near zone being smaller than the one of the top part. This is illustrated by the right image of Figure 1.

When spherical coordinates are used in ophthalmic optics, the center of coordinates is referenced at the center of the eye. Using this model, the angle of rotation of the eye is the angle of rotation of the model, and the point with radius 0 (with any angle) is the center of the eye. An example of this use of spherical coordinates can be seen in Figure 1.21 of [7] and Figure 2. The left part of Figure 2 shows the lateral view of a progressive surface with the spherical coordinates centered at the center of the eye. In the right part of Figure 2 we can see that the point corresponds to a point of the far region. 


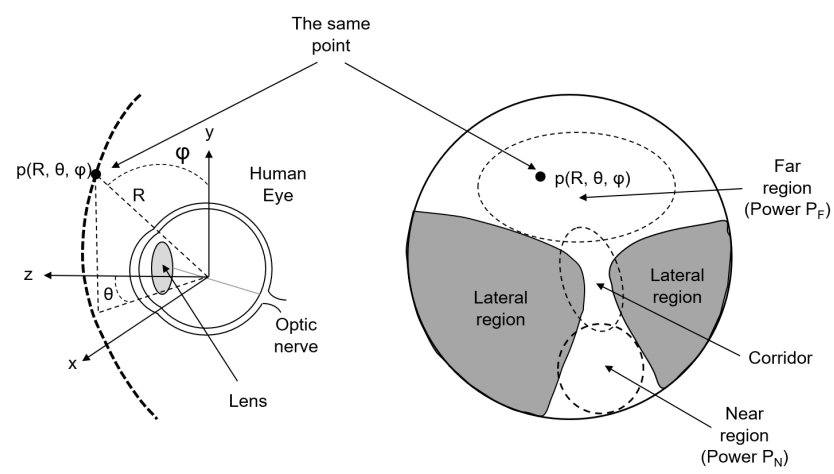

Fig. 2 Example of spherical coordinates centered at the center of the eye.

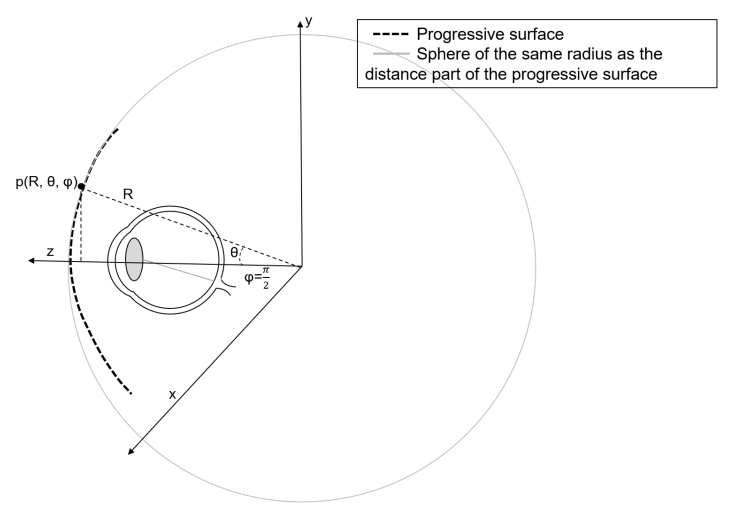

Fig. 3 Example of spherical coordinates centered at the center of the sphere corresponding to the far region.

However, the model proposed in this article does not have any relation to this model.

As stated above, the far zone of a progressive lens can be approximated by a sphere, because in this region the power of the lens and consequently its curvature is nearly a constant. The center of this sphere will be the center of the new proposed model in spherical coordinates. One angle is in the normal direction of the lens, while the other angle is in the perpendicular direction. The main motivation for this new model is to have better convexity properties than previous existing models. The representation of this new model is shown in Figure 3. In this image, the radius is near a constant $R$ for all the points of the far region; the radius is smaller for the points of the near region. The radius will vary gradually from far region to near region.

The literature about the calculation of progressive lenses is scarce, mainly for reasons of confidentiality, since it is a problem solved in the private indus- 
try. However, there are a few available references. In [3] the use of B-splines and a Cartesian model was suggested. The work [4] was a follow-up of [3] and introduced a gradient model for this problem. In [6] the optimization problem was shown to be nonconvex, and in [12] multilevel nonlinear optimization methods for this problem were developed, which were able to compute a progressive lens in approximately 20 minutes. Although it was not stated, the model described in [6] was in Cartesian coordinates. In fact, the term Cartesian is not used in any of the previous references, because it is implicit. Another line of research is described in [7, where the surface of the lens is modeled using Zernike's polynomials and interior point optimization techniques.

The quality of the solution obtained using spherical coordinates will in general be the same than that of the Cartesian coordinates. The main advantage of the spherical coordinates is that theoretically the problem is "less nonconvex" (as it will be shown), and consequently the optimization problem is ideally expected to converge faster. The data provided in the model using spherical coordinates has to be referenced in angles (radians), and the data provided by the model using Cartesian coordinates has to be referenced in distances $(\mathrm{mm})$ in the projected $x-y$ plane.

The structure of this paper is as follows. The definition and computation of the power and astigmatism, and the optic model of the problem is given in Section 2 for Cartesian coordinates, and in Section 3 for spherical coordinates. In these sections we also provide some discussion about convexity issues. Section 4 introduces the optimization model and Section 5 provides numerical results using the interior point solvers LOQO, IPOPT and KNITRO hooked to AMPL. Section 5 also shows examples of progressive lenses computed using the new model. The (tedious) computation of the power and astigmatism using spherical coordinates is shown in Appendix A.

\section{Power and astigmatism in Cartesian coordinates}

\subsection{Definition and computation of power and astigmatism}

The goal of this article is to build a progressive surface having (1) the minimum astigmatism (aberrations); (2) the requested power in the far region; (3) the requested power in the near region; (4) and such that the astigmatism in the corridor, in the far, and in the near regions must be less than a certain threshold value. As stated in the introduction, the astigmatism corresponds to the aberrations, and it must be minimized. By power we mean the optical power of the lens, which must be equal to some predefined value for the far region and another bigger value for the near region. The main parameters for the model are thus the powers of the far and near regions, the shape of these regions, and the maximum levels of astigmatism allowed in the far region, corridor and near region.

The power and the astigmatism of a lens are defined as follows: 
Definition 1 Let be $k_{1}$ and $k_{2}$ the two principal curvatures at a given point of a surface, then

$$
\begin{aligned}
\text { Astigmatism } & =(\mu-1)\left|k_{1}-k_{2}\right|, \\
\text { Power } & =(\mu-1) \frac{k_{1}+k_{2}}{2},
\end{aligned}
$$

where $\mu$ is the refraction index of the material.

If we express the curvatures in $m^{-1}$ (inverse of meters), then the astigmatism and the power are expressed in diopters $\left(1 D=1 \mathrm{~m}^{-1}\right)$.

The principal curvatures $k_{1}$ and $k_{2}$ at a given point of the surface of the lens will be computed considering the following general parameterization of the lens:

$$
\begin{aligned}
\mathbb{R}^{2} & \longmapsto \mathbb{R}^{3} \\
(u, v) & \longmapsto \vec{p}(u, v)=(x(u, v), y(u, v), z(u, v)) .
\end{aligned}
$$

From differential geometry [2,8,9], $k_{1}$ and $k_{2}$ are the eigenvalues of the Weingarten application defined by the matrix

$$
A=\left(\begin{array}{ll}
e & f \\
f & g
\end{array}\right)\left(\begin{array}{ll}
E & F \\
F & G
\end{array}\right)^{-1}
$$

where $E, F, G$ and $e, f, g$ are the coefficients of the first and second fundamental form. They are expressed as

$$
\begin{array}{lll}
E=\overrightarrow{p_{u}} \cdot \overrightarrow{p_{u}} & F=\overrightarrow{p_{u}} \cdot \overrightarrow{p_{v}} & G=\overrightarrow{p_{v}} \cdot \overrightarrow{p_{v}} \\
e=\vec{n} \cdot \overrightarrow{p_{u u}} & f=\vec{n} \cdot \overrightarrow{p_{u v}} & g=\vec{n} \cdot \overrightarrow{p_{v v}},
\end{array}
$$

where $\cdot$ is the dot product, $\vec{n}$, defined as

$$
\vec{n}=\frac{\overrightarrow{p_{u}} \times \overrightarrow{p_{v}}}{\left|\overrightarrow{p_{u}} \times \overrightarrow{p_{v}}\right|}
$$

is the normal vector to the surface $(x$ being the cross or vector product of two vectors in $\mathbb{R}^{3}$ ), and the subscripts $u, v$ denote the partial derivative respect to $u$ or $v$, that is,

$$
\overrightarrow{p_{u}}=\left(\frac{\partial x}{\partial u}, \frac{\partial y}{\partial u}, \frac{\partial z}{\partial u}\right) \quad \overrightarrow{p_{v}}=\left(\frac{\partial x}{\partial v}, \frac{\partial y}{\partial v}, \frac{\partial z}{\partial v}\right)
$$

The second-order partial derivatives are thus

$\overrightarrow{p_{u u}}=\left(\frac{\partial^{2} x}{\partial u^{2}}, \frac{\partial^{2} y}{\partial u^{2}}, \frac{\partial^{2} z}{\partial u^{2}}\right), \overrightarrow{p_{u v}}=\left(\frac{\partial^{2} x}{\partial u \partial v}, \frac{\partial^{2} y}{\partial u \partial v}, \frac{\partial^{2} z}{\partial u \partial v}\right), \overrightarrow{p_{v v}}=\left(\frac{\partial^{2} x}{\partial v^{2}}, \frac{\partial^{2} y}{\partial v^{2}}, \frac{\partial^{2} z}{\partial v^{2}}\right)$.

From (3), $A$ is a $2 \times 2$ matrix, which can be rewritten as

$$
A=\left(\begin{array}{ll}
a_{1,1} & a_{1,2} \\
a_{2,1} & a_{2,2}
\end{array}\right)
$$


and then its eigenvalues can be computed by

$$
P(\lambda)=\operatorname{det}(A-\lambda I)=\left|\begin{array}{cc}
a_{1,1}-\lambda & a_{1,2} \\
a_{2,1} & a_{2,2}-\lambda
\end{array}\right|=\lambda^{2}-\operatorname{tr}(A) \lambda+\operatorname{det}(A),
$$

where

$$
\begin{aligned}
\operatorname{tr}(A) & =a_{1,1}+a_{2,2} \\
\operatorname{det}(A) & =a_{1,1} a_{2,2}-a_{1,2} a_{2,1}
\end{aligned}
$$

are respectively the trace and the determinant of the matrix $A$. The solutions to $P(\lambda)=0$ are

$$
\lambda=\frac{\operatorname{tr}(A) \pm \sqrt{\operatorname{tr}(A)^{2}-4 \operatorname{det}(A)}}{2}=\frac{\operatorname{tr}(A)}{2} \pm \sqrt{\left(\frac{\operatorname{tr}(A)}{2}\right)^{2}-\operatorname{det}(A)} .
$$

Denoting $K=\operatorname{det}(A)$ and $H=\frac{1}{2} \operatorname{tr}(A), k_{1}$ and $k_{2}$ are defined as

$$
k_{1}, k_{2}=H \pm \sqrt{\left(H^{2}-K\right)}
$$

where $H$ and $K$ are named the mean and Gaussian curvature, respectively. From (3) we get

$$
\begin{aligned}
& K=\operatorname{det}(A)=\frac{e g-f^{2}}{E G-F^{2}} \\
& H=\frac{1}{2} \operatorname{tr}(A)=\frac{e G-2 f F+g E}{2\left(E G-F^{2}\right)}
\end{aligned}
$$

So we have a method to calculate the power and astigmatism at each point of the lens.

2.2 Computing power and astigmatism using Cartesian coordinates

From (2), considering $x(u, v)=u, y(u, v)=v$ and using Cartesian coordinates, the surface of a lens can be defined as a function

$$
\begin{aligned}
& z: \mathbb{R}^{2} \longrightarrow \mathbb{R} \\
& (x, y) \rightarrow z(x, y) .
\end{aligned}
$$

The main advantage of using Cartesian coordinates is that the expressions $\overrightarrow{p_{u}}, \overrightarrow{p_{v}}, \overrightarrow{p_{u u}}, \overrightarrow{p_{u v}}$ and $\overrightarrow{p_{v v}}$ are simplified. The first-order partial derivatives at a given point in Cartesian coordinates are

$$
\overrightarrow{p_{u}}=\left(1,0, \frac{\partial z(x, y)}{\partial x}\right), \overrightarrow{p_{v}}=\left(0,1, \frac{\partial z(x, y)}{\partial y}\right),
$$

while the second-order partial derivatives are given by

$\overrightarrow{p_{u u}}=\left(0,0, \frac{\partial^{2} z(x, y)}{\partial x^{2}}\right), \overrightarrow{p_{u v}}=\left(0,0, \frac{\partial^{2} z(x, y)}{\partial x \partial y}\right), \overrightarrow{p_{v v}}=\left(0,0, \frac{\partial^{2} z(x, y)}{\partial y^{2}}\right)$ 
The normal vector to the surface is then

$$
\vec{n}=\frac{\overrightarrow{p_{u}} \times \overrightarrow{p_{v}}}{\left|\overrightarrow{p_{u}} \times \overrightarrow{p_{v}}\right|}=\frac{1}{\sqrt{1+\left(\frac{\partial z}{\partial x}\right)^{2}+\left(\frac{\partial z}{\partial y}\right)^{2}}} .
$$

From (4), 12, 13, 14, and (15) the mean curvature and Gaussian curvature are respectively

$$
H(x, y)=\frac{\frac{\partial^{2} z}{\partial x^{2}}\left(1+\frac{\partial z}{\partial y}\right)^{2}-2 \frac{\partial^{2} z}{\partial x \partial y} \frac{\partial z}{\partial x} \frac{\partial z}{\partial y}+\frac{\partial^{2} z}{\partial y^{2}}\left(1+\frac{\partial z}{\partial x}\right)^{2}}{2\left(1+\left(\frac{\partial z}{\partial x}\right)^{2}+\left(\frac{\partial z}{\partial y}\right)^{2}\right)^{3 / 2}}=\frac{1}{2}\left(k_{1}+k_{2}\right)
$$

and

$$
K(x, y)=\frac{\frac{\partial^{2} z}{\partial x^{2}} \frac{\partial^{2} z}{\partial y^{2}}-\left(\frac{\partial^{2} z}{\partial x \partial y}\right)^{2}}{\left(1+\left(\frac{\partial z}{\partial x}\right)^{2}+\left(\frac{\partial z}{\partial y}\right)^{2}\right)}=k_{1} k_{2}
$$

Consequently,

$$
\begin{array}{r}
\operatorname{Pow}(x, y)=(\mu-1) H(x, y) \\
\operatorname{Ast}(x, y)=-2(\mu-1) \sqrt{H(x, y)^{2}-K(x, y)}
\end{array}
$$

So we have calculated the power and astigmatism in Cartesian coordinates.

\subsection{Example: a sphere}

We consider a thin lens (without center thickness) whose surface is a part of a sphere of radius $R$. We will calculate the power and the astigmatism of the sphere using previous formulas. The parameterization in Cartesian coordinates is

$$
\begin{aligned}
& z: \mathbb{R}^{2} \longrightarrow \mathbb{R} \\
& (x, y) \rightarrow z(x, y)=-\sqrt{R^{2}-x^{2}-y^{2}} .
\end{aligned}
$$

The previous formulation corresponds to a sphere of radius $R$ centered at $(0,0,0)$. As we consider the negative root, we take into account the inferior part of the sphere.

Using (17), 18), 19) and (20) and calculating the first and second partial derivatives of 21) we finally obtain

$$
\begin{array}{rlrl}
\operatorname{Pow}(x, y) & =\frac{\mu-1}{R} & & \forall(x, y): x^{2}+y^{2} \leq R^{2} \\
\text { Ast }(x, y) & =0 & \forall(x, y): x^{2}+y^{2} \leq R^{2} .
\end{array}
$$

This means that a sphere of radius $R$ has zero astigmatism and constant power $\frac{\mu-1}{R}$ for all the surface. For example, if we want to get a sphere of power $5 \mathrm{D}$ using a refraction index of the material of 1.6 , we need a radius $(\mu-1) / 5=(1.6-1) / 5=0.12 \mathrm{~m}=120 \mathrm{~mm}$. 
2.4 Nonconvexity in Cartesian coordinates

The expressions of power and astigmatism expressed in Cartesian coordinates are (in general) clearly nonlinear and, when used in the formulation of the constraints of an optimization problem, give rise to a nonconvex set of solutions and a nonconvex feasible region (this was also noted in [6]). To illustrate this fact, let us first consider the following Example 1, which corresponds to a lens with $P_{F}=P_{N}$ (i.e., with the same power in far and near regions).

Example 1 Given the function (defined on a grid of points in $\mathbb{R}^{2}$ )

$$
\begin{aligned}
z: G \subseteq \mathbb{R}^{2} & \longrightarrow \mathbb{R} \\
\left(x_{i}, y_{i}\right) & \longrightarrow z\left(x_{i}, y_{i}\right) \quad i=1, \ldots, n,
\end{aligned}
$$

we find the surface $z(x, y)$ solution to the following problem:

$$
\begin{aligned}
& \min _{z\left(x_{i}, y_{i}\right)} 1 \\
& \text { subject to } \\
& \operatorname{Ast}\left(x_{i}, y_{i}\right) \quad=0 \\
& P-\epsilon \leq \operatorname{Pow}\left(x_{i}, y_{i}\right) \quad \leq P+\epsilon \quad \forall\left(x_{i}, y_{i}\right) \in G \\
& z(0,0) \quad=-\frac{1-\mu}{P} \\
& \left.\frac{\partial z(x, y)}{\partial x}\right|_{x=0, y=0}=0 \\
& \left.\frac{\partial z(x, y)}{\partial y}\right|_{x=0, y=0} ^{x=0, y=0}=0,
\end{aligned}
$$

where $\operatorname{Pow}\left(x_{i}, y_{i}\right)$ and $A s t\left(x_{i}, y_{i}\right)$ are defined in $170-207, P=5 D, \epsilon=0.12 D$, $\mu=1.6$, and the points $\left(x_{i}, y_{i}\right)$ satisfy $-45 \mathrm{~mm} \leq x_{i}, y_{i} \leq 45 \mathrm{~mm}$.

The solution to 23 is a set of spheres of radius $R$ centered at point $(0,0,0)$, such that $R_{\min } \leq R \leq R_{\max }$ where

$$
\begin{aligned}
& R_{\text {min }}=(\mu-1) /(R+\epsilon)=(1.6-1) /(5+0.12)=0.11719 m=117.19 \mathrm{~mm} \\
& R_{\text {max }}=(\mu-1) /(R-\epsilon)=(1.6-1) /(5-0.12)=0.12295 \mathrm{~m}=122.95 \mathrm{~mm} .
\end{aligned}
$$

Considering two different solutions of (23)

$$
\begin{aligned}
& \text { Solution } 1: z^{1}\left(x_{i}, y_{i}\right)=-\sqrt{R_{\text {min }}^{2}-x_{i}^{2}-y_{i}^{2}} \\
& \text { Solution } 2: z^{2}\left(x_{i}, y_{i}\right)=-\sqrt{R_{\text {max }}^{2}-x_{i}^{2}-y_{i}^{2}}
\end{aligned}
$$

we observe that

$$
a z^{1}\left(x_{i}, y_{i}\right)+b z^{2}\left(x_{i}, y_{i}\right) \neq-\sqrt{R_{k}^{2}-x_{i}^{2}-y_{i}^{2}} \quad \forall R_{k} \in \mathbb{R}, \text { where } a+b=1 .
$$

Therefore the solution set (an thus also the feasible region) of (23) (in Cartesian coordinates) is not a convex set. However, with another parameterization (in particular, using spherical coordinates) the feasible region of that problem becomes convex. This new parameterization is shown in the next section. 


\section{Power and astigmatism in spherical coordinates}

3.1 Model with spherical coordinates

The model using spherical coordinates is

$$
\begin{aligned}
\mathbb{R}^{2} & \longrightarrow \mathbb{R}^{3} \\
(\theta, \varphi) & \longmapsto \vec{p}(\theta, \varphi)
\end{aligned}
$$

where

$$
\vec{p}=\left(\begin{array}{l}
x(\theta, \varphi) \\
y(\theta, \varphi) \\
z(\theta, \varphi)
\end{array}\right)=\left(\begin{array}{c}
R(\theta, \varphi) \cos (\theta) \\
R(\theta, \varphi) \sin (\theta) \cos (\varphi) \\
R(\theta, \varphi) \sin (\theta) \sin (\varphi)
\end{array}\right) \quad \text { and } \quad \theta, \varphi \in[0, \pi]
$$

Considering a grid $\left(\theta_{i}, \varphi_{j}\right), i=1, \ldots, n, j=1, \ldots, n$ where each $\theta_{i} \in[0, \pi]$ and each $\varphi_{j} \in[0, \pi]$, we get

$$
\begin{aligned}
x_{i j} & =R_{i j} \cos \left(\theta_{j}\right) \\
y_{i j} & =R_{i j} \sin \left(\theta_{j}\right) \cos \left(\varphi_{i}\right) \\
z_{i j} & =R_{i j} \sin \left(\theta_{j}\right) \sin \left(\varphi_{i}\right),
\end{aligned}
$$

$R_{i j}$ being the variables, and $\theta_{i}$ and $\varphi_{j}$ the parameters of the model.

It is worth remarking that we also considered the alternative model (a rotation of (28)):

$$
\begin{aligned}
& x(\theta, \varphi)=R(\theta, \varphi) \sin (\theta) \cos (\varphi) \\
& y(\theta, \varphi)=R(\theta, \varphi) \sin (\theta) \sin (\varphi) \\
& z(\theta, \varphi)=R(\theta, \varphi) \cos (\theta) \\
& \text { and } \quad \theta \in\left[0, \frac{\pi}{2}\right] \\
& \varphi \in[-\pi, \pi] \text {, }
\end{aligned}
$$

which was discarded because it had some singularities in the center of the lens when calculating the power and the astigmatism of a progressive lens.

The expressions for power and astigmatism using model (27) are given in Appendix A

\subsection{Convexity in spherical coordinates}

As shown by next Proposition 1, the spherical coordinates model exhibits better convexity properties than the Cartesian model of Section 2.

Proposition 1 Using the spherical coordinates model of Subsection 3.1, the feasible region of the optimization problem formulated in Example 1 -which was not convex-becomes a convex set. 
Proof Given the function

$$
\begin{aligned}
R: G \subseteq \mathbb{R}^{2} & \longrightarrow \mathbb{R} \\
\left(\theta_{i}, \varphi_{i}\right) & \longrightarrow R\left(\theta_{i}, \varphi_{i}\right) \quad i=1, \ldots, n,
\end{aligned}
$$

and the spherical coordinates defined by (27), we find the radii $R\left(\theta_{i}, \varphi_{i}\right)$ solution to the following optimization problem:

$$
\begin{aligned}
& \min _{R\left(\theta_{i}, \varphi_{i}\right)} 1 \\
& \text { subject to } \\
& \operatorname{Ast}\left(\theta_{i}, \varphi_{i}\right) \quad= \\
& \begin{array}{rlrl}
P-\epsilon \leq & P \text { Pow }\left(\theta_{i}, \varphi_{i}\right) & \leq P+\epsilon & \forall\left(\theta_{i}, \varphi_{i}\right) \in G \\
R\left(\frac{\pi}{2}, \frac{\pi}{2}\right) & =-\frac{1-\mu}{P} &
\end{array} \\
& \left.\frac{\partial R\left(\theta_{i}, \varphi_{i}\right)}{\partial \theta_{i}}\right|_{\theta_{i}=\frac{\pi}{2}, \varphi_{i}=\frac{\pi}{2}}=0 \\
& \left.\frac{\partial R\left(\theta_{i}, \varphi_{i}\right)}{\partial \varphi_{i}}\right|_{\theta_{i}=\frac{\pi}{2}, \varphi_{i}=\frac{\pi}{2}}=0,
\end{aligned}
$$

where $\operatorname{Ast}\left(\theta_{i}, \varphi_{i}\right)$ and $\operatorname{Pow}\left(\theta_{i}, \varphi_{i}\right)$ are defined in 51) and 52 in Appendix A

Using the same data as in (23) (from Example 1), that is, $P=5 D, \epsilon=$ $0.12 D, \mu=1.6$, and $\left(\theta_{i}, \varphi_{i}\right)$ is a grid of angles, where $0 \leq \theta_{i}, \varphi_{i} \leq \pi$, the solution of $(30)$ is a set of spheres of radius $R$ centered at point $(x=0, y=$ $0, z=0)$, such that $R_{\min } \leq R \leq R_{\max }$, where

$$
\begin{aligned}
& R_{\min }=(\mu-1) /(R+\epsilon)=(1.6-1) /(5+0.12)=0.11719 m=117.19 m m \\
& R_{\max }=(\mu-1) /(R-\epsilon)=(1.6-1) /(5-0.12)=0.12295 m=122.95 \mathrm{~mm} .
\end{aligned}
$$

Considering two different solutions of 30 :

$$
\begin{array}{cr}
\text { Solution } 1: R^{1}\left(\theta_{i}, \varphi_{i}\right)=R_{\min } & \forall \theta_{i} \in[0, . ., \pi] \\
\text { Solution 2: } R^{2}\left(\theta_{i}, \varphi_{i}\right)=R_{\max } & \forall \varphi_{i} \in[0, . ., \pi],
\end{array}
$$

we have

$$
\begin{array}{r}
a R^{1}\left(\theta_{i}, \varphi_{i}\right)+b R^{2}\left(\theta_{i}, \varphi_{i}\right)=R\left(\theta_{i}, \varphi_{i}\right) \quad \text { for some } R \in\left[R_{\min }, R_{\max }\right] \\
\text { where } a+b=1 \text { and } a, b \geq 0
\end{array}
$$

thus proving that the solution set is convex.

The spherical coordinates model will be used in the following sections to compute progressive lenses. In the next sections progressive lenses will be calculated from scratch, as done in an industrial environment.

\section{The optimization model}

The goal of the optimization problem is to obtain a square surface with certain optical properties. The optical properties are the astigmatism and the power, as defined in previous sections. Predefined values of power and maximum values of astigmatism will be imposed in certain regions of the surface. In addition, the surface has to be as smooth as possible, with the minimum levels of astigmatism. These two last conditions will be controlled by the objective function. 
Parameters of the model

The main parameters for the definition of the model are:

$-\left(\theta_{i}, \varphi_{j}\right) \in[0, \pi] \times[0, \pi],(i, j) \in \mathcal{G}=\{1, \ldots, n\} \times\{1, \ldots, n\}$, is a grid of angles (in radians) used for the definition of the lens in spherical coordinates, where $n$ denotes the number of angles for each dimension of the grid. The grid is defined such that $\left(\theta_{\frac{1+n}{2}}, \varphi_{\frac{1+n}{2}}\right)=\left(\frac{\pi}{2}, \frac{\pi}{2}\right)$. The grid $\mathcal{G}$ is partitioned in three subsets: $\mathcal{G}=\mathcal{F} \cup \mathcal{N} \cup \mathcal{B} . \mathcal{F}^{2}$ and $\mathcal{N}$ are the set of angles in the far and near regions of the lens, respectively, where some values of power will be imposed; $\mathcal{B}$ is the set of remaining angles, corresponding to regions of the lens whose power will not be constrained.

$-\left(\theta_{i^{\prime}}^{\prime}, \varphi_{j^{\prime}}^{\prime}\right) \in[0, \pi] \times[0, \pi],\left(i^{\prime}, j^{\prime}\right) \in \mathcal{G}^{\prime}=\{1, \ldots, o\} \times\{1, \ldots, o\}$, is another grid of angles (radians), with $\mathcal{G}^{\prime}$ much coarser than $\mathcal{G}$ (i.e., $o \ll n$ ), where $o$ is the number of angles used in the definition of a B-Spline whose coefficients are the variables of the optimization model (see next Section).

- $P_{F}$ and $P_{N}$ are the requested powers (in diopters) in the far and near regions of the lens, respectively $\left(P_{N}>P_{F}\right)$.

$-T$ is a tolerance expressed in meters that appears when bounding the variable radius.

$-\mu \in[1.5,1.9]$ is refraction index of the material of the lens.

- The subset $\mathcal{F}$ of far region angles of the grid is partitioned in $k$ additional far subregions $\mathcal{F}=\mathcal{F}_{1} \cup \cdots \cup \mathcal{F}_{k}$. For each of these $k$ subregions we consider a tolerance $\epsilon_{h}, h=1, \ldots, k$, of the soft constraints for the power (in diopters). The total number of far region constraints is then $\sum_{h=1}^{k}\left|\mathcal{F}_{h}\right|$.

- Similarly to the far region, the set of angles $\mathcal{N}$ of the near region is partitioned in $l$ near subregions $\mathcal{N}=\mathcal{N}_{1} \cup \cdots \cup \mathcal{N}_{l}$. For each of these $l$ subregions we consider a tolerance $\delta_{h}, h=1, \ldots, l$, of the soft constraints for the power (in diopters). The total number of near region constraints is $\sum_{h=1}^{l}\left|\mathcal{N}_{h}\right|$.

- The grid $\mathcal{G}$ of angles is also partitioned in different $m$ subregions of astigmatism, that is, $\mathcal{G}=\mathcal{A}_{1} \cup \cdots \cup \mathcal{A}_{m}$. An upper bound $\beta_{h}, h=1, \ldots, m$ will be imposed to the astigmatism (diopters) of angles in each subregion. The total number of astigmatism constraints is $\sum_{h=1}^{m}\left|\mathcal{A}_{h}\right|$.

- Finally, $w_{1}, w_{2}, w_{3} \in[0,1] \subset \mathbb{R}$ are weights of the different parts of the objective function (defined below in (35)).

Variables of the model

The variables of the optimization problem are the coefficients of a three-degree B-spline surface, as defined in [11, page 100]. These coefficients are denoted as

$$
\mathbb{R} \ni c\left(\theta_{i^{\prime}}^{\prime}, \varphi_{j^{\prime}}^{\prime}\right) \geq 0, \quad\left(i^{\prime}, j^{\prime}\right) \in \mathcal{G}^{\prime} .
$$

Using the B-spline we define the radius of the surface for the grid $\mathcal{G}$ as

$$
R\left(\theta_{i}, \varphi_{j}\right)=\sum_{i^{\prime}=1}^{o} \sum_{j^{\prime}=1}^{o} c\left(\theta_{i^{\prime}}^{\prime}, \varphi_{j^{\prime}}^{\prime}\right) B_{i^{\prime}}^{3}\left(\theta_{i}\right) B_{j^{\prime}}^{3}\left(\varphi_{j}\right), \quad(i, j) \in \mathcal{G},
$$


where $B_{i^{\prime}}^{3}\left(\theta_{i}\right)$ and $B_{j^{\prime}}^{3}\left(\varphi_{j}\right),(i, j) \in \mathcal{G},\left(i^{\prime}, j^{\prime}\right) \in \mathcal{G}^{\prime}$, are the 1-dimensional threedegree B-splines basis defined in [11, page 100].

From the spherical coordinates equations (27), we can compute the power and the astigmatism for all the surface of the lens (i.e. for points $(i, j) \in \mathcal{G}$ ) using the formulae (34) and (41)-(53). In particular, (51) and (52) provide the definition of power and astigmatism respectively. We remark that the definition of $R(\theta, \varphi)$ as a B-spline allows us to calculate its derivatives using (41)- 43].

The optimization problem has only $o^{2}$ variables, but the surface can be evaluated in $n^{2}$ points (where $o \ll n$ ). Indeed, this is the main reason for the use of a B-spline in the model.

Another property of the three-degree B-spline is that it is a two times continuously differentiable surface and three times differentiable (the third derivative is not continuous, and the fourth derivative is zero). This means that the radius defined in (34) allows us to compute its first, second and third derivatives. The first and second derivatives are needed to calculate the power and the astigmatism, for all the $n^{2}$ points of the grid $\mathcal{G}$. The power and astigmatism are thus continuous, although they are only evaluated in a grid of $n^{2}$ points. The third degree of differentiability allows us to compute the gradients of the astigmatism and power, which will be needed in the below objective function (35).

Four-, five-, and six-degree B-splines have been tested in order to increase the quality of the gradient of the astigmatism and power, and consequently the quality of the solution. The even degrees four and six did not work correctly. The five-degree B-splines worked as well as the three-degree B-splines, but required between 2.6 and 4.8 more computational time. Therefore three-degree B-splines have been used.

\section{Objective function}

The objective function (35) consists of the minimization of the sum of the squared astigmatism and squared norm of the gradients of power and astigmatism, for all the points of the grid $\mathcal{G}$. These factors are weighted by $w_{1}, w_{2}, w_{3} \in$ $[0,1]$. The objective function is:

$$
\begin{aligned}
\min \sum_{(i, j) \in \mathcal{G}} \frac{1}{n^{2}}( & w_{1}\left(A s t\left(\theta_{i}, \varphi_{j}\right)\right)^{2}+ \\
& w_{2}\left(\left(\frac{\partial A s t\left(\theta_{i}, \varphi_{j}\right]}{\partial \theta}\right)^{2}+\left(\frac{\partial A s t\left(\theta_{i}, \varphi_{j}\right)}{\partial \varphi}\right)^{2}\right)+ \\
& \left.w_{3}\left(\left(\frac{\partial \operatorname{Pow}\left(\theta_{i}, \varphi_{j}\right)}{\partial \theta}\right)^{2}+\left(\frac{\partial \operatorname{Pow}\left(\theta_{i}, \varphi_{j}\right)}{\partial \varphi}\right)^{2}\right)\right),
\end{aligned}
$$

where $n$ is the number of angles in each dimension of the grid. 
Constraints

The objective function is minimized subject to the following three groups of constraints:

$$
\begin{array}{r}
P_{F}-\epsilon_{h} \leq \operatorname{Pow}\left(\theta_{i}, \varphi_{j}\right) \leq P_{F}+\epsilon_{h}(i, j) \in \mathcal{F}_{h}, h=1, \ldots, k, \\
P_{N}-\delta_{h} \leq \operatorname{Pow}\left(\theta_{i}, \varphi_{j}\right) \leq P_{N}+\delta_{h}(i, j) \in \mathcal{N}_{h}, h=1, \ldots, l, \\
\operatorname{Ast}\left(\theta_{i}, \varphi_{j}\right)^{2} \leq \beta_{h}^{2}(i, j) \in \mathcal{A}_{h}, h=1, \ldots, m .
\end{array}
$$

These constraints define the philosophy of the design of the progressive lens. Constraints (36) and (37) control the power in the different far and near subregions of the lens, while constraints 38 fix a maximum of astigmatism in certain regions of the lens. Constraints (38) are squared to avoid the square root in the definition of the astigmatism (52), making the model simpler. The quality and characteristics of the progressive lens is governed by the values of the parameters $\epsilon_{h}, \delta_{h}$ and $\beta_{h}$, and the sets $\mathcal{F}_{h}, \mathcal{N}_{h}$ and $\mathcal{A}_{h}$; setting the proper values is the most difficult part in terms of optics.

A second set of three constraints impose conditions in the midpoint of the grid $\left(\frac{\pi}{2}, \frac{\pi}{2}\right)$ (and on the lens surface):

$$
\begin{array}{ll}
R\left(\frac{\pi}{2}, \frac{\pi}{2}\right) & =\frac{1-\mu}{P_{F}} \\
\left.\frac{\partial R\left(\theta_{i}, \varphi_{i}\right)}{\partial \theta}\right|_{\theta_{i}=\frac{\pi}{2}, \varphi_{i}=\frac{\pi}{2}} & =0 \\
\left.\frac{\partial R\left(\theta_{i}, \varphi_{i}\right)}{\partial \varphi}\right|_{\theta_{i}=\frac{\pi}{2}, \varphi_{i}=\frac{\pi}{2}} & =0 .
\end{array}
$$

The purpose of these three constraints is to center the lens in the three dimensional space: the first one imposes a certain radius, while the other two guarantee it to be perpendicular to the normal of the surface. These are the only equality constraints of the model.

The last set of constraints are the bounds of variables $R\left(\theta_{i}, \varphi_{i}\right)$ and a bound of the power, for all the surface of the lens:

$$
\begin{aligned}
& R\left(\theta_{i}, \varphi_{i}\right) \leq-\frac{1-\mu}{P_{F}}+T(i, j) \in \mathcal{G} \\
& R\left(\theta_{i}, \varphi_{i}\right) \geq-\frac{1-\mu}{P_{N}}-T(i, j) \in \mathcal{G} \\
& \operatorname{Pow}\left(\theta_{i}, \varphi_{j}\right) \geq P_{F}(i, j) \in \mathcal{G}
\end{aligned}
$$

The first two groups of constraints of 40 bound the feasible region and were helpful for the convergence of the optimization solver (but they are not compulsory and inactive in the optimal solution). The last group of constraints of (40 impose a minimum value of power in all the points of the lens.

Finally, the optimization problem to be solved is the minimization of (35), subject to constraints (36), (37), 38), 39, (40) and (34). 


\section{Numerical results}

\subsection{Problem instances}

We generated a set of 15 problem instances, denoted as P1, P2,..,P15, obtained with different sets of parameters. However, some parameters are common for all the 15 problems, such as:

$-P_{F}=5 \mathrm{D}$ (power in the far region).

$-P_{N}=7 \mathrm{D}$ (power in the near region).

$-T=0.045 \mathrm{~m}$ (tolerance of bound 40 ).

$-\mu=1.6$ (index of refraction of the lens material).

$-n=61\left(n^{2}\right.$ being the number of angles of the grid $\left.\mathcal{G}\right)$.

$-o=30\left(o^{2}\right.$ being the number of angles of $\mathcal{G}^{\prime}$, the grid used for the definition of the B-splines). We remind that the B-splines can be evaluated at any angle, not only at the list of $o^{2}$ angles. In particular, for each problem we have $n^{2}=61^{2}=3721$ points where the B-splines can be evaluated.

- $\left(\theta_{i}, \varphi_{j}\right),(i, j) \in \mathcal{G}$ (the particular angles used in the grid $\left.\mathcal{G}\right)$.

- $\left(\theta_{i}^{\prime}, \varphi_{j}^{\prime}\right),\left(i^{\prime}, j^{\prime}\right) \in \mathcal{G}^{\prime}$ (the particular angles used in the grid $\mathcal{G}^{\prime}$ ).

We remark that:

- the subset $\mathcal{F}$ of far region angles and its partition in $k$ far subregions $\mathcal{F}=\mathcal{F}_{1} \cup \cdots \cup \mathcal{F}_{k}$,

- the subset $\mathcal{N}$ of near region angles and its partition in $l$ near subregions $\mathcal{N}=\mathcal{N}_{1} \cup \cdots \cup \mathcal{N}_{l}$,

- and the partition of $m$ subregions of astigmatism $\mathcal{G}=\mathcal{A}_{1} \cup \cdots \cup \mathcal{A}_{m}$,

are different for each problem.

The grid of angles $\mathcal{G}$ is computed by the formula $\theta_{i}=\varphi_{i}=0.9817477042+$ $0.01963495(i-1) \mathrm{rad}$, for $i=1, \ldots, n$ (where $n=61)$. Expressing the angles in degrees we have $\theta_{i}=\varphi_{i}=33.75+1.125(i-1)^{\circ}$, for $i=1, \ldots, n$. For example, $\theta_{1}=\varphi_{1}=0.9817477042 \mathrm{rad}\left(\right.$ or $\left.33.75^{\circ}\right) ; \theta_{31}=\varphi_{31}=1.5707963268=\frac{\pi}{2} \mathrm{rad}$ (or $\left.90^{\circ}\right)$; and $\theta_{61}=\varphi_{61}=2.1598449493 \mathrm{rad}\left(\right.$ or $\left.123.75^{\circ}\right)$.

Among the parameters that differ for each problem we find $\epsilon_{h} h=1, \ldots, k$, $\delta_{h} h=1, \ldots, l$, and $\beta_{h} h=1, \ldots, m$.

\section{Optimization problem for a particular instance (P5)}

Let us consider a particular instance, e.g., P5. For this problem we have $k=4$ far regions, $l=3$ near regions, and $m=11$ astigmatism regions. Tolerances $\epsilon_{h}, h=1, \ldots, 4$, of far region constraints 36 are, respectively, 0.03, 0.06, 0.12 and 0.25 . For near region constraints 37 , tolerances $\delta_{h}, h=1,2,3$ are 0.03 , $0.12,0.25$. Finally, the 11 tolerances $\beta_{h}$ for astigmatism constraints (38) were $0.03,0.12,0.25,0.03,0.06,0.12,0.10,0.15,0.20,0.25,0.06$. These tolerances are expressed in diopters $(\mathrm{D})$.

The four, three and 11 respectively far, near and astigmatism regions (defined by sets $\mathcal{F}_{h}, \mathcal{N}_{h}$, and $\mathcal{A}_{h}$ ) are shown in Figures 4,5 and 6 , using different 


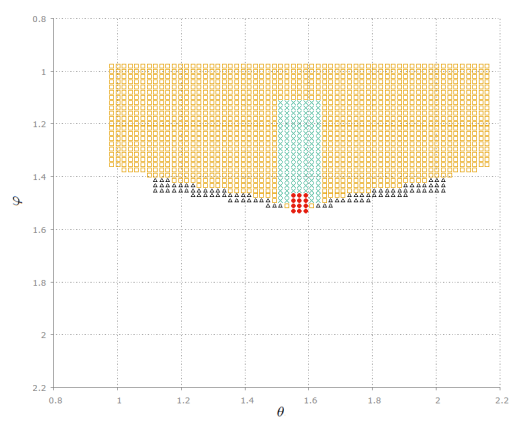

Fig. 4 The four far regions of problem P5, each in a different color and pattern.

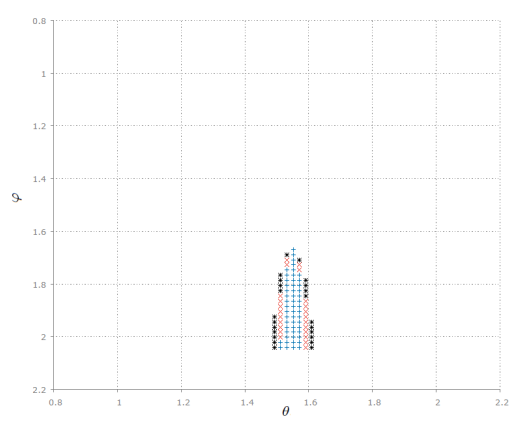

Fig. 5 The three near regions of problem P5, each in a different color and pattern.

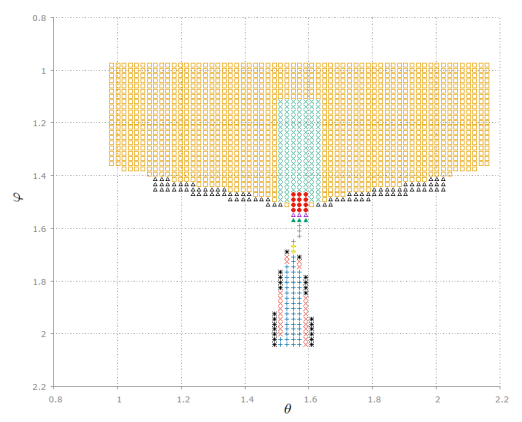

Fig. 6 The 11 astigmatism regions of problem P5, each in a different color and pattern.

colors and patterns for each subregion. From Figure 5 we see that the near regions are concentric; this fact, together with the values of $\delta_{h}$ and the objective function, guarantee that the change in power will be gradual, obtaining a smoother lens. This same behaviour also applies to far and astigmatism regions of Figures 4 and 6 . In Figure 6 we also observe that some astigmatism 


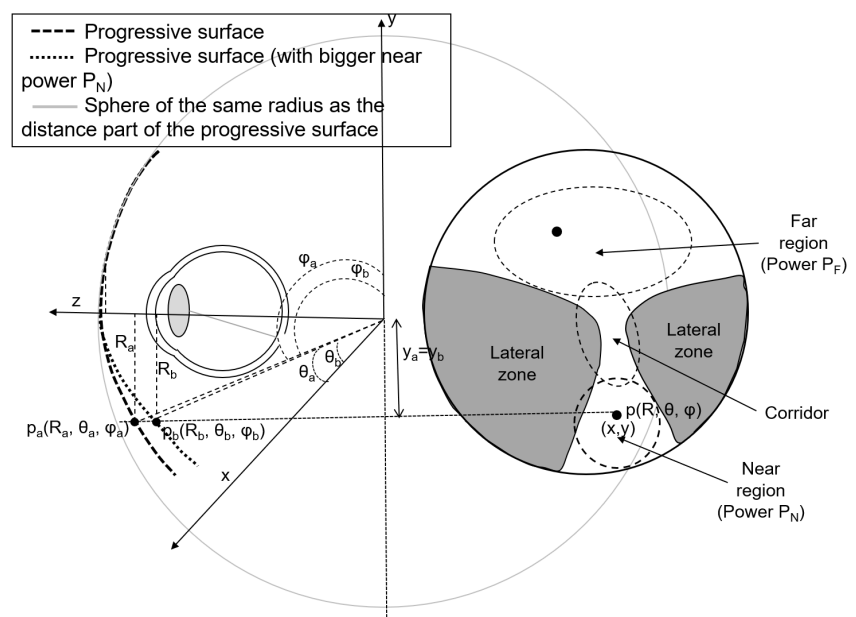

Fig. 7 Conversion of $(x, y)$ near point from Cartesian to spherical coordinates.

regions correspond to the part of the lens named corridor, which connects the far and near regions.

\section{Expressing the far, near and astigmatism regions in angles}

Far, near and astigmatism regions are defined using the grids $\mathcal{F}_{h}, \mathcal{N}_{h}$, and $\mathcal{A}_{h}$ of angles. Unfortunately, optical progressive lenses designers have the information about those grids in Cartesian coordinates (in $\mathrm{mm}$ ). In a Cartesian model this is not a problem, since constraints can be expressed in points $(x, y)$. But in our spherical model this information about the regions has to be converted to angles $(\theta, \varphi)$. The conversion from $(x, y)$ to $(\theta, \varphi)$ depends on $R(\theta, \varphi)$ as can be seen in (27). As $R(\theta, \varphi)$ is the solution of our problem, we have an issue to solve.

For instance, consider the near region in Cartesian coordinates $(\mathrm{mm})$ of the right image of Figure 7, which corresponds to the set

$$
\left\{(x, y) \in \mathbb{R}^{2}:(x-3)^{2}+(y+15)^{2} \leq 5^{2}\right\},
$$

that is, a circle of radius $5 \mathrm{~mm}$ centered at point $(3 \mathrm{~mm},-15 \mathrm{~mm})$. Converting this region into spherical coordinates is not an easy task. We will focus on the conversion of the particular point $(x, y)$ in the near region of the right image of Figure 7. The left image of the figure shows two progressive lenses with the same $P_{F}$ (power in far region) and different $P_{N}$ (power in near region). It can be seen in this left image that the conversion of point $(x, y)$ from Cartesian to spherical coordinates depends on the shape of the lens, and also on $P_{F}$ and $P_{N}$. In this figure, point $(x, y)$ has two different projections in the $\mathbb{R}^{3}$ space in spherical coordinates. In the image we can appreciate that $\theta_{a} \neq \theta_{b}$ because $\vec{p}_{a} \neq \vec{p}_{b}$. This means that the grids of angles (for far, near and 
astigmatism regions), which are parameters of the optimization model, depend on the solution of the problem (the $R\left(\theta_{i}, \varphi_{j}\right)$ ). In order to solve this issue, what is done is to pre-calculate a progressive lens of the same $P_{F}$ and $P_{N}$ (and $\mu$ ), to obtain approximate values of radius for the far and near regions; the $(x, y)$ points are thus converted from Cartesian to spherical coordinates using this approximate radius, and the inverse of equation (27). Using this technique, we can convert all the (far, near and astigmatism) regions from Cartesian to spherical coordinates.

In the case of this work, as $P_{F}, P_{N}$ and $\mu$ were the same for all the 15 instances, we remark that once the radius in far and near regions were approximated, these approximations could be used for all the 15 problems. The radius considered for the corridor region was approximated by the mean of the radius of the far and near regions. The complete procedure for the generation and solution of the 15 instances was thus as follows:

- Firstly, compute a single progressive lens fixing $P_{F}, P_{N}$ and $\mu$ using Cartesian coordinates.

- Compute an approximated radius for the far and near regions.

- Using these radius, compute all the far, near and astigmatism regions for instances P1, P2, P3, . , P15 in spherical coordinates, considering different tolerances for power in far and near regions, and astigmatism.

- Solve the 15 instances using the spherical coordinates model.

Improving the instance generator would be one the aspects to deal with in a future work.

\section{Objective function}

The objective function (35) was used for all the 15 instances, using different weights $w_{1}, w_{2}$, and $w_{3}$ :

$-w_{1}=0, w_{2}=0, w_{3}=0$, for problems P1 and P2 (that is, the objective function is a constant).

$-w_{1}=1, w_{2}=0, w_{3}=0$, for problems P3, P4 and P5.

$-w_{1}=0, w_{2}=1, w_{3}=0$, for problems P6, P7 and P8.

$-w_{1}=0, w_{2}=0, w_{3}=1$, for problems P9, P10 and P11.

$-w_{1}=1, w_{2}=1, w_{3}=0$, for problems P12, P13.

- $w_{1}=1, w_{2}=0, w_{3}=1$, for problems P14 and P15.

In order to simplify our problem we did not use weights others than 0 or 1 . Using other weights in the objective function makes difficult the comprehension of the results in the optimal solution. The units of the objective function are $D^{2}$ for problems P3, P4 P5; $\frac{D^{2}}{\operatorname{rad}^{2}}$ for problems P6, P7, P8, P9, P10, P11; and the sum of $D^{2}$ and $\frac{D^{2}}{\mathrm{rad}^{2}}$ for problems P12, P13, P14 and P15.

Finally, Table 11 reports the number of constraints for the 15 instances generated. The number of variables is always the same, $o^{2}=30^{2}=900$, which is the number of points in the grid $\mathcal{G}^{\prime}$. In general we have around 16000 (nonlinear) constraints, and most of them are inequalities. 


\begin{tabular}{lc}
\hline Problem & n. of constraints \\
\hline P1 & 18057 \\
P2 & 15870 \\
P3 & 15870 \\
P4 & 15936 \\
P5 & 15870 \\
P6 & 15836 \\
P7 & 15836 \\
P8 & 15836 \\
P9 & 15870 \\
P10 & 15836 \\
P11 & 15836 \\
P12 & 15870 \\
P13 & 15936 \\
P14 & 15870 \\
P15 & 15836 \\
\hline
\end{tabular}

Table 1 Number of constraints for each problem. The number of variables was always 900

\subsection{Computational environment}

The optimization model was implemented using the AMPL modeling language [5] linked with three different interior points solvers: LOQO [13], KNITRO [1] and IPOPT 14. Due to our availability of licenses (for AMPL, LOQO and KNITRO, which are commercial products), two different servers were used. The first one has eight 2.7GHz AMD Opteron 8384 Shanghay CPUs, with 32 cores and $128 \mathrm{~GB}$ RAM. This computer has the AMPL modeling language installed as well as the LOQO 6.0.6 and IPOPT 3.8.1 solvers. The second machine was a Fujitsu Primergy RX300, with two $3.33 \mathrm{GHz}$ Intel Xeon X5680 CPUs, with 24 cores and 144GB RAM. The AMPL modeling language, and the solvers KNITRO 10.1.0, IPOPT 3.9.3 and IPOPT 3.12.8 are installed in this second server. Both servers will be referred to as "server 1" and "server 2" in the following sections.

\subsection{Stopping criteria}

Firstly, we used one of the solvers (LOQO was the choice) with different stopping criteria in order to decide which tolerances at the optimum are sufficient to get a good quality progressive lens. The quality of the lens must be evaluated in terms of optics, analyzing the isolines of the optimal lens as well as the value of the objective function.

We ran the 15 problems using five different stopping criteria with LOQO, obtained by adjusting the tolerances sigfig (the number of equal digits in the primal and dual objective functions) and inftol (infeasibility tolerance for the primal and dual problems). Table 2 reports the values of the primal and dual objective functions at the last iteration for problem P12. We chose problem $\mathrm{P} 12$ because the objective function is affected by the square of the astigmatism 


\begin{tabular}{lrrr}
\hline Stopping criteria & primal o.f. & dual o.f. & relative error \\
\hline sigfig $=2$, inftol $=10^{-3}$ & 10.14186825 & -1257.362782 & 124.9774321 \\
sigfig $=4$, inftol $=10^{-6}$ & 1.634879469 & 1.395858085 & 0.146201227 \\
sigfig $=6$, inftol $=10^{-3}$ & 1.62718076 & 1.580678331 & 0.028578527 \\
sigfig $=8$, inftol $=10^{-3}$ & 1.626118778 & 1.625710862 & 0.000250853 \\
sigfig $=8$, inftol $=10^{-12}$ & 1.626114492 & 1.626114214 & $1.7096 \mathrm{E}-07$ \\
\hline
\end{tabular}

Table 2 Primal objective value, dual objective value and relative error at the optimum, using LOQO 6.0.6 and five different stopping criteria for problem P12.
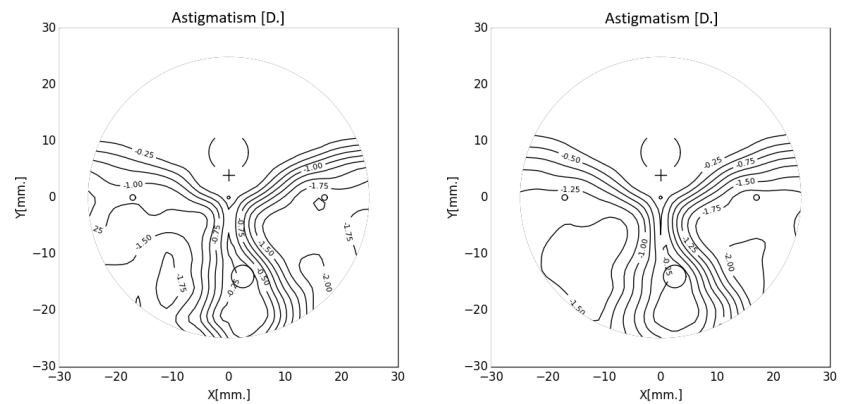

Fig. 8 Astigmatism of the lens of P12 using LOQO 6.0.6 and two different stopping criteria: sigfig $=2$, inftol $=10^{-3}$ (left) and sigfig $=4$, inftol $=10^{-6}$ (right).

\begin{tabular}{lcccc}
\hline Problem & $\begin{array}{c}\text { sigfig } 4 \\
\text { inftol } 10^{-6}\end{array}$ & $\begin{array}{c}\text { sigfig } 6 \\
\text { inftol } 10^{-3}\end{array}$ & $\begin{array}{c}\text { sigfig } 8 \\
\text { inftol } 10^{-3}\end{array}$ & $\begin{array}{c}\text { sigfig } 8 \\
\text { inftol } 10^{-12}\end{array}$ \\
\hline P1 & 313 & 34 & 124 & 411 \\
P2 & 385 & 29 & 30 & 461 \\
P3 & 54 & 50 & 69 & 87 \\
P4 & 60 & 53 & 86 & 94 \\
P5 & 54 & 50 & 69 & 87 \\
P6 & 36 & 55 & 109 & 131 \\
P7 & 95 & 62 & 126 & 160 \\
P8 & 36 & 55 & 109 & 131 \\
P9 & 41 & 84 & 105 & 115 \\
P10 & 33 & 49 & 63 & 68 \\
P11 & 41 & 44 & 55 & 60 \\
P12 & 42 & 48 & 63 & 74 \\
P13 & 41 & 44 & 57 & 69 \\
P14 & 36 & 44 & 58 & 65 \\
P15 & 36 & 48 & 64 & 68 \\
\hline
\end{tabular}

Table 3 Number of iterations for each problem with LOQO 6.0.6 and different stopping criteria.

as well as its partial derivatives. The relative error (last column of Table 2 is defined as: |primal o.f. - dual o.f.|/|primal o.f.|.

Numerically, from first line of Table 2, we conclude that the first stopping condition sigfig $=2$, inftol $=10^{-3}$ has not enough quality. In order to evaluate the solutions in terms of the optical properties of the lens produced, Figure 

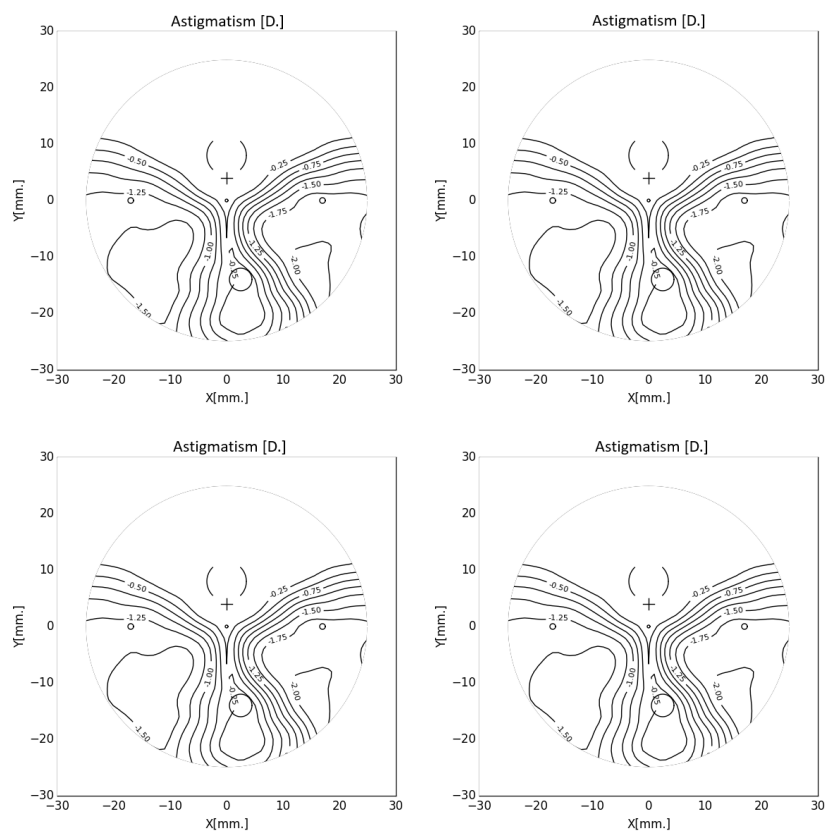

Fig. 9 Astigmatism of the lens of problem P12 using LOQO 6.0.6 and four different stopping criteria: sigfig $=4$, inftol $=10^{-6}$ (top left); sigfig $=6$, inftol $=10^{-3}$ (top right); sigfig $=8$, inftol $=10^{-3}$ (bottom left); and sigfig $=8$, inftol $=10^{-12}$ (bottom right).

8 shows the astigmatism map for the lens obtained using LOQO and two different stopping criteria; the units of these maps are diopters (D) for the isolines and the axes $x$ and $y$ are displayed in $\mathrm{mm}$. We see that left image ( igfig $=2$, inftol $\left.=10^{-3}\right)$ is blurrier than the right one $\left(\right.$ sigfig $=4$, inftol $\left.=10^{-6}\right)$. From an optics perspective, the use of sigfig $=4$, inftol $=10^{-6}$ is preferred in order to get a good quality lens.

Figure 9 shows the lenses obtained with the four last stopping criteria of Table 2 (i.e., the tighter ones). We observe that the four lenses obtained are of similar quality, and then consequently the stopping criteria that solves the problem faster (in terms of number of iterations, and thus also in terms of seconds) will be preferred. Table 3 shows the number of iterations required for all the 15 instances and the four stopping criteria. Considering only P12 we would choose the stopping condition sigfig $=4$, inftol $=10^{-6}$, but for P1 and $\mathrm{P} 2$, where the objective function is constant, we see that the fastest executions were obtained with sigfig $=6$, inftol $=10^{-3}$. We remark that problems P1 and P2 using sigfig $=4$, inftol $=10^{-6}$ required a large number of iterations to converge.

We concluded that for LOQO 6.06, the most suitable stopping criteria were sigfig $=6$ and inftol $=10^{-3}$. Since each solver might have different parameters or tolerances, for the rest of solvers we chose those which are closer to sigfig $=$ 6 and inftol $=10^{-3}$, as shown below. 


\subsection{Solvers comparison}

From now on we will consider six different combinations of solvers and servers:

- LOQO 6.0.6, server 1.

- IPOPT 3.8.1, server 1.

- IPOPT 3.9.3, server 2.

- IPOPT 3.12.8, server 2.

- KNITRO 10.1.0 with interior point/direct algorithm (algorithm 1), server 2.

- KNITRO 10.1.0 with interior point/conjugate gradient algorithm (algorithm 2), server 2 .

The two variants of KNITRO differ in how the Newton's equation is solved at each iteration of the interior point algorithm, either by a direct method (factorization), or through an iterative conjugate gradient [1]. IPOPT and LOQO use a direct method for this step [13,14.

The stopping conditions used for each solver within AMPL were:

- LOQO 6.0.6: sigfig $=4$, inftol $=10^{-6}$.

- IPOPT (all versions): tol $=10^{-2}$.

- KNITRO 10.1.0 (both algorithms 1 and 2): opttol $=10^{-3}$.

All the solvers reported the solutions obtained as either "optimal" (LOQO and IPOPT) or "locally optimal" or "satisfactory solution" (KNITRO). It is worth noting that, although the problem is nonlinear and nonconvex, and then each solver could provide a different local minima, when visualizing the obtained lenses we observed that the six solutions found for each problem were the same (except for negligible numerical differences).

Table 4 shows the number of iterations for the 15 problems and six solvers. The number of iterations for LOQO and IPOPT were between 29 and 84, while KNITRO exhibited a larger variability: it performed between 21 and 376 iterations with the direct algorithm 1, and between 18 and 158 with the conjugate gradient algorithm 2. That is, in some cases KNITRO was the best solver (for example for instances P2 and P15) but it was the worst in others (for example for P12). The CPU time, reported in Table 5. was proportional to the number of iterations. We remind that the first two solvers (LOQO and IPOPT 3.8.1) were executed in server 1 and the other four in server 2 ; this explains the different times between the first IPOPT version and the other two, while all of them had similar number of iterations (server 2 was on average 2.8 times faster than server 1 ).

In order to evaluate the solutions, not only in terms of the optical properties of the lens produced, but also in terms of optimization, we checked the objective functions and constraints at optimal points. The objective functions are showed in Table 6. We see that in general KNITRO with algorithm 2 (conjugate gradient) provides the largest objectives (which is not surprising since conjugate gradient is meant to approximately solve the Newton's equations); the lowest objectives are provided by LOQO and KNITRO with algorithm 1 


\begin{tabular}{ccccccc}
\hline Problem & $\begin{array}{c}\text { LOQO } \\
\text { IP.0.6 }\end{array}$ & $\begin{array}{c}\text { IPOP } \\
3.8 .1\end{array}$ & $\begin{array}{c}\text { IPOPT } \\
3.12 .8\end{array}$ & $\begin{array}{c}\text { IPOPT } \\
3.9 .3\end{array}$ & $\begin{array}{c}\text { KNITRO } \\
10.1 .0 \text { alg 1 }\end{array}$ & $\begin{array}{c}\text { KNITRO } \\
10.1 .0 \text { alg 2 }\end{array}$ \\
\hline P1 & 34 & 80 & 80 & 79 & 150 & 24 \\
P2 & 29 & 39 & 39 & 39 & 21 & 18 \\
P3 & 50 & 44 & 44 & 44 & 36 & 22 \\
P4 & 53 & 56 & 56 & 56 & 62 & 22 \\
P5 & 50 & 44 & 44 & 44 & 36 & 22 \\
P6 & 55 & 51 & 51 & 51 & 198 & 53 \\
P7 & 62 & 46 & 46 & 46 & 376 & 48 \\
P8 & 55 & 51 & 51 & 51 & 198 & 53 \\
P9 & 84 & 49 & 49 & 49 & 53 & 25 \\
P10 & 49 & 54 & 54 & 54 & 49 & 39 \\
P11 & 44 & 51 & 51 & 51 & 53 & 34 \\
P12 & 48 & 52 & 52 & 52 & 261 & 43 \\
P13 & 44 & 66 & 66 & 66 & 262 & 26 \\
P14 & 44 & 50 & 50 & 50 & 47 & 26 \\
P15 & 48 & 51 & 51 & 51 & 48 & \\
\hline
\end{tabular}

Table 4 Number of iterations for each problem using the six different solvers.

\begin{tabular}{|c|c|c|c|c|c|c|}
\hline Problem & $\begin{array}{c}\text { LOQO } \\
6.0 .6 \\
\text { (server } 1 \text { ) } \\
\end{array}$ & $\begin{array}{c}\text { IPOPT } \\
3.8 .1 \\
\text { (server 1) } \\
\end{array}$ & $\begin{array}{c}\text { IPOPT } \\
3.12 .8 \\
\text { (server 2) } \\
\end{array}$ & $\begin{array}{c}\text { IPOPT } \\
3.9 .3 \\
\text { (server 2) } \\
\end{array}$ & $\begin{array}{c}\text { KNITRO } \\
10.1 .0 \text { alg } 1 \\
\text { (server } 2 \text { ) }\end{array}$ & $\begin{array}{c}\text { KNITRO } \\
10.1 .0 \text { alg } 2 \\
(\text { server } 2)\end{array}$ \\
\hline$\overline{\mathrm{P} 1}$ & 112 & 211 & 90 & 93 & 128 & 51 \\
\hline $\mathrm{P} 2$ & 115 & 122 & 63 & 73 & 51 & 65 \\
\hline P3 & 165 & 134 & 66 & 75 & 60 & 65 \\
\hline $\mathrm{P} 4$ & 163 & 158 & 75 & 78 & 78 & 66 \\
\hline P5 & 165 & 135 & 74 & 68 & 62 & 64 \\
\hline P6 & 688 & 562 & 200 & 169 & 598 & 327 \\
\hline $\mathrm{P} 7$ & 769 & 512 & 184 & 158 & 1094 & 314 \\
\hline $\mathrm{P} 8$ & 616 & 562 & 201 & 174 & 601 & 325 \\
\hline P9 & 925 & 522 & 191 & 154 & 184 & 163 \\
\hline P10 & 565 & 539 & 219 & 172 & 179 & 295 \\
\hline P11 & 462 & 512 & 182 & 164 & 192 & 250 \\
\hline P12 & 616 & 578 & 204 & 180 & 852 & 861 \\
\hline P13 & 572 & 718 & 244 & 207 & 774 & 278 \\
\hline P14 & 472 & 510 & 179 & 161 & 170 & 187 \\
\hline $\mathrm{P} 15$ & 561 & 549 & 182 & 164 & 174 & 179 \\
\hline
\end{tabular}

Table 5 Number of seconds for each problem using the six different solvers.

(direct solver); and IPOPT objectives were in between. Last column of Table 6 reports for each problem the difference between the minimum and the maximum objective functions obtained divided by the objective function of LOQO (taken as a baseline). The largest of these ratios was 0.73 for P5 (and P3). Figure 10 shows the maps of power and astigmatism for P5 using LOQO, KNITRO with direct algorithm 1, and KNITRO with conjugate gradient algorithm 2; we observe that these three lenses are quite the same in terms of optics. However, the lens obtained using KNITRO with conjugate gradient algorithm 2 is a little bit different around the point $(x=22.5 \mathrm{~mm}, y=5 \mathrm{~mm})$, and it is also the lens with a larger objective function. We remark that the differences of the values of the objective functions are not significant in terms 


\begin{tabular}{|c|c|c|c|c|c|c|c|}
\hline $\begin{array}{l}\text { Pro- } \\
\text { blem }\end{array}$ & $\begin{array}{c}\text { LOQO } \\
6.0 .6\end{array}$ & $\begin{array}{c}\text { IPOPT } \\
3.8 .1 \\
\end{array}$ & $\begin{array}{c}\text { IPOPT } \\
3.12 .8 \\
\end{array}$ & $\begin{array}{c}\text { IPOPT } \\
3.9 .3\end{array}$ & $\begin{array}{c}\text { KNITRO } \\
10.1 .0 \mathrm{alg} 1\end{array}$ & $\begin{array}{c}\text { KNITRO } \\
10.1 .0 \text { alg } 2\end{array}$ & $\begin{array}{c}\text { Max. diff. } \\
\text { (relative } \\
\text { LOQO) }\end{array}$ \\
\hline P1 & 1.0000 & 1.0000 & 1.0000 & 1.0000 & 1.0000 & 1.0000 & 0.00 \\
\hline $\mathrm{P} 2$ & 1.0000 & 1.0000 & 1.0000 & 1.0000 & 1.0000 & 1.0000 & 0.00 \\
\hline P3 & 0.2575 & 0.2824 & 0.2824 & 0.2824 & 0.2566 & 0.4455 & 0.73 \\
\hline $\mathrm{P} 4$ & 0.8872 & 0.9113 & 0.9113 & 0.9113 & 0.8859 & 1.0057 & 0.14 \\
\hline P5 & 0.2575 & 0.2824 & 0.2824 & 0.2824 & 0.2566 & 0.4455 & 0.73 \\
\hline P6 & 0.9106 & 0.9234 & 0.9234 & 0.9234 & 0.9100 & 1.1671 & 0.28 \\
\hline P7 & 0.4348 & 0.4494 & 0.4494 & 0.4494 & 0.4354 & 0.6841 & 0.57 \\
\hline P8 & 0.9106 & 0.9234 & 0.9234 & 0.9234 & 0.9100 & 1.1671 & 0.28 \\
\hline P9 & 15.5478 & 15.5529 & 15.5529 & 15.5529 & 15.5434 & 16.1208 & 0.04 \\
\hline P10 & 11.8692 & 11.8786 & 11.8786 & 11.8786 & 11.8733 & 11.8832 & 0.00 \\
\hline P11 & 12.1851 & 12.1957 & 12.1957 & 12.1957 & 12.1815 & 12.1842 & 0.00 \\
\hline P12 & 1.6272 & 1.6470 & 1.6470 & 1.6470 & 1.6261 & 1.6304 & 0.01 \\
\hline P13 & 2.9763 & 2.9892 & 2.9892 & 2.9892 & 2.9739 & 3.3132 & 0.11 \\
\hline P14 & 16.9738 & 16.9763 & 16.9763 & 16.9763 & 16.9709 & 16.9802 & 0.00 \\
\hline $\mathrm{P} 15$ & 13.9853 & 13.9923 & 13.9923 & 13.9923 & 13.9826 & 14.2659 & 0.02 \\
\hline
\end{tabular}

Table 6 Objective function for each problem using the six different solvers.

of optical properties of the lens. The difference of the isolines around the point $(x=22.5 \mathrm{~mm}, y=5 \mathrm{~mm})$ do not affect the quality of the lens. We can conclude that the solutions using any of the six different solvers are the same in terms of optics.

To check the (primal feasibility of the) constraints we will focus again on the maps of astigmatism and power of instance P5 in Figure 10. We note that, in the far, corridor, and near regions, the power and the astigmatism have the required values. In addition, the maximum and the minimum for all the lens for the astigmatism and for the power are also in accordance with the constraints (36)-(38). The astigmatism for all the points is smaller than $1.2 \cdot 2.0=2.4 D$ and the power is between $5 D$ and $7 D$. A similar behaviour was observed for the rest of problems (whose maps of astigmatism and power are not reported here to save space).

We finally remark that, using exactly the same stopping conditions (opttol $=10^{-3}$ ), KNITRO with direct algorithm 1 and conjugate gradient algorithm 2 did not produce the same results: it was faster and worse with algorithm 2 than with algorithm 1. Again this can be explained because conjugate gradient solves approximately the Newton direction at each interior point iteration. Using KNITRO with the active set algorithm (algorithm 3) and the SQP algorithm (algorithm 4) the problems did not converge.

To sum up, we were able to solve 15 different instances with six different solvers with the spherical coordinates model; and in all cases we obtained high quality progressive lenses. As said before, all the lenses obtained were equivalent for the six solvers. 

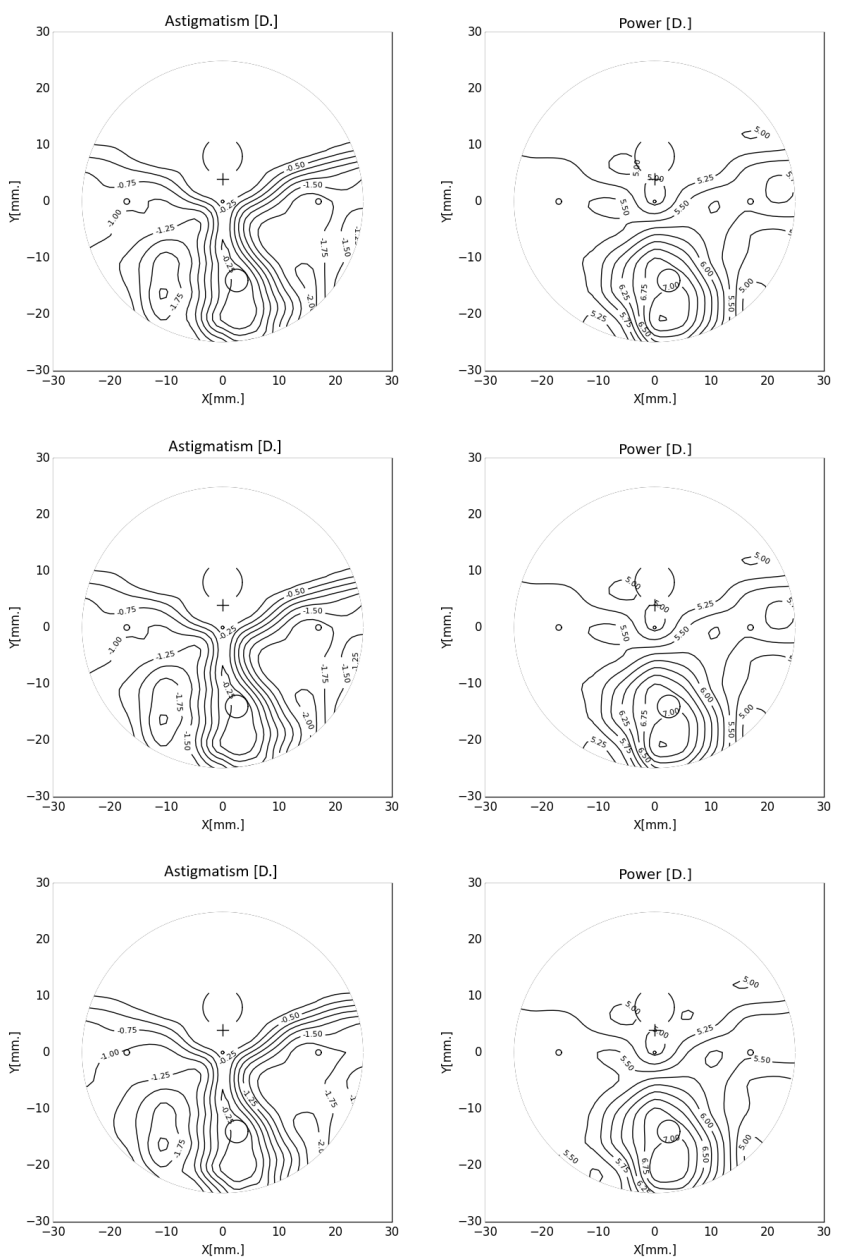

Fig. 10 Astigmatism (left) and power (right) of the lens of problem P5 using LOQO 6.0.6 (top), KNITRO with direct algorithm 1 (middle) and KNITRO with conjugate gradient algorithm 2 (bottom).

\section{Conclusions}

We described a new model using spherical coordinates and its implementation allowed us to compute real-world progressive lenses in a successful way. The progressive lenses obtained have a good quality in terms of optics and are similar to the progressive lenses obtained using previously existing Cartesian coordinates models. In addition, they are the same quality as other progressive lenses that are for sale. However, we remark that these lenses are not the same design: the lenses obtained using spherical coordinates usually have the corridor a little bit longer, and may have the far region or the near region bigger 
or smaller than the lenses obtained using Cartesian coordinates. In the market of progressive lenses, all of these lenses are accepted. We did not succeed to get an equivalence between the data required to calculate the lenses using Cartesian coordinates and the data required when using spherical coordinates. Such an equivalence is only possible if the lens are solved in both systems of coordinates. The main advantage of using spherical coordinates is that in theory the problem exhibits better convexity properties.

When comparing the different solvers we observed that LOQO and IPOPT have a small variability in terms of number of iterations (and consequently in CPU time). This was not observed for KNITRO using the direct and conjugate gradient algorithms: in some instances it converged faster than the others solvers, while in others it took much more time and iterations. For instance, in four out the 15 cases KNITRO with direct algorithm converged faster - in number of iterations - than LOQO; in two cases performed the same number of iterations; and in nine cases it was outperformed by LOQO. Although the value of the objective functions obtained in the optimum, as well as the value of the variables are not exactly the same for all the solvers, the differences between the solutions are not significant in terms of optics, and they can be considered equivalent.

A further task to be done would be to enhance the relation between the data used in the model with Cartesian coordinates and the data used in the model with spherical coordinates. Enhancing this relation would improve the quality of the instance generator.

\section{Acknowledgments}

This work has been supported by the Industrial Doctorate grant 2014-DI-023 of the Agència de Gestió d'Ajuts Universitaris i de Recerca of the Government of Catalonia.

\section{References}

1. R.H. Byrd, J. Nocedal, R.A. Waltz. Feasible interior methods using slacks for nonlinear optimization, Computational Optimization and Applications, 26, 35-61, 2003.

2. M.P. de Carmo. Differential Geometry of Curves and Surfaces. Prentice-Hall, Inc., 1976, New Jersey, USA.

3. J.C. Dursteler. Computer Assisted Design Systems for Progressive Lenses (in Spanish). PhD thesis, Universitat Politècnica de Catalunya, 1991.

4. E. Fontdecaba. High Performance Algorithms for Progressive Addition Lens Design. $\mathrm{PhD}$ thesis, Universitat Politècnica de Catalunya, 2000.

5. R. Fourer, D.M. Gay, D.W. Kernighan. AMPL: A Modeling Language for Mathematical Programming. Duxbury Press, Pacific Grove CA, USA, 2002.

6. N. Gould, Ph. Toint. How Mature is Nonlinear Optimization?, in Applied Mathematics Entering the 21st Century: Invited Talks from the ICIAM 2003 Congress, (J. H. Hill and R. Moore, eds.) SIAM, Philadelphia, pp. 141-161, 2004.

7. X. Jonsson. Méthodes de Points Intérieurs et de Régions de Confiance en Optimisation Non Linéaire. Application à la Conceptions de Verrers Ophtalmiques Progressifs. $\mathrm{PhD}$ thesis, Université Paris 6, 2002. 
8. C. Horneber, M. Knauer, G. Häusler. Phase measuring deflectomery - a new method to measure reflecting surfaces, p.7, Annual Report, Optik 2000.

9. M. Lipschutz. Differential Geometry. Schaum's, McGraw-Hill,1969.

10. G. Minkwitz, Uber den flachenastigmatismus bei gewissen symmetrischen aspharen, Optica Acta: International Journal of Optics, 10, 223-227, 1963.

11. L. Piegel, W. Tiller. The Nurbs Book. Springer, 1997, Berlin.

12. D. Tomanos. Algorithms and Software for Multilevel Nonlinear Optimization. PhD thesis, University of Namur (FUNDP), Namur, 2009.

13. R.J. Vanderbei. LOQO user's manual-version 3.10, Optimization Methods and Software, 11, 485-514, 1999.

14. A. Wächter, L.T. Biegler. On the implementation of a primal-dual interior point filter line search algorithm for large-scale nonlinear programming, Mathematical Programming 106, 25-57, 2006.

\section{A Calculus of power and astigmatism of a surface using spherical coordinates}

This section provides the expressions of the power and astigmatism of the surface defined using the spherical coordinates (27).

Firstly, the first and second derivatives of $\vec{p}(\theta, \varphi)$ need to be calculated:

$$
\begin{aligned}
& \overrightarrow{p_{\theta}}=\left(\begin{array}{c}
\frac{\partial R(\theta, \varphi)}{\partial \theta} \cos (\theta)-R(\theta, \varphi) \sin (\theta) \\
\frac{\partial R(\theta, \varphi)}{\partial \theta} \sin (\theta) \cos (\varphi)+R(\theta, \varphi) \cos (\theta) \cos (\varphi) \\
\frac{\partial R(\theta, \varphi)}{\partial \theta} \sin (\theta) \sin (\varphi)+R(\theta, \varphi) \cos (\theta) \sin (\varphi)
\end{array}\right)^{\top} \\
& \overrightarrow{p_{\varphi}}=\left(\begin{array}{c}
\frac{\partial R(\theta, \varphi)}{\partial \varphi} \cos (\theta) \\
\frac{\partial R(\theta, \varphi)}{\partial \varphi} \sin (\theta) \cos (\varphi)-R(\theta, \varphi) \sin (\theta) \sin (\varphi) \\
\frac{\partial R(\theta, \varphi)}{\partial \varphi} \sin (\theta) \sin (\varphi)+R(\theta, \varphi) \sin (\theta) \cos (\varphi)
\end{array}\right)^{\top} \\
& \overrightarrow{p_{\theta \theta}}=\left(\begin{array}{c}
\frac{\partial^{2} R(\theta, \varphi)}{\partial \theta^{2}} \cos (\theta)+2 \frac{\partial R(\theta, \varphi)}{\partial \theta} \sin (\varphi)-R(\varphi, \theta) \cos (\varphi) \\
\frac{\partial^{2} R(\theta, \varphi)}{\partial^{2} \partial \theta^{2}} \sin (\theta) \cos (\varphi)+2 \frac{\partial R(\theta, \varphi)}{\partial \theta} \cos (\theta) \cos (\varphi)-R(\theta, \varphi) \sin (\theta) \cos (\varphi) \\
\frac{\partial^{2} R(\theta, \varphi)}{\partial \theta^{2}} \sin (\theta) \sin (\varphi)+2 \frac{\partial R(\theta, \varphi)}{\partial \theta} \cos (\theta) \sin (\varphi)-R(\theta, \varphi) \sin (\theta) \sin (\varphi)
\end{array}\right)^{\top}, \\
& \overrightarrow{p_{\theta \varphi}}=\left(\begin{array}{c}
\frac{\partial^{2} R(\theta, \varphi)}{\partial \theta \partial \varphi} \cos (\theta)-\frac{\partial R(\theta, \varphi)}{\partial \varphi} \sin (\theta) \\
\frac{\partial^{2} R(\theta, \varphi)}{\partial \theta \partial \varphi} \sin (\theta) \cos (\varphi)+\frac{\partial R(\theta, \varphi)}{\partial \varphi} \cos (\theta) \cos (\varphi)-\frac{\partial R(\theta, \varphi)}{\partial \theta} \sin (\theta) \sin (\varphi)-R(\theta, \varphi) \cos (\theta) \sin (\varphi) \\
\frac{\partial^{2} R(\theta, \varphi)}{\partial \theta \partial \varphi} \sin (\theta) \sin (\varphi)+\frac{\partial R(\theta, \varphi)}{\partial \varphi} \cos (\theta) \sin (\varphi)+\frac{\partial R(\theta, \varphi)}{\partial \theta} \sin (\theta) \cos (\varphi)+R(\theta, \varphi) \cos (\theta) \cos (\varphi)
\end{array}\right)^{\top}, \\
& \overrightarrow{p_{\varphi \varphi}}=\left(\begin{array}{c}
\frac{\partial^{2} R(\theta, \varphi)}{\partial \varphi^{2}} \cos (\theta) \\
\frac{\partial^{2} R(\theta, \varphi)}{\partial \varphi^{2}} \sin (\theta) \sin (\varphi)-2 \frac{\partial R(\theta, \varphi)}{\partial \varphi} \sin (\theta) \cos (\varphi)-R(\theta, \varphi) \sin (\theta) \cos (\varphi) \\
\frac{\partial^{2} R(\theta, \varphi)}{\partial \varphi^{2}} \sin (\theta) \sin (\varphi)+2 \frac{\partial R(\theta, \varphi)}{\partial \varphi} \sin (\theta \cos (\varphi)-R(\theta, \varphi) \sin (\theta) \sin (\varphi)
\end{array}\right)^{\top}
\end{aligned}
$$

The coefficients of the first and second fundamental forms $E, F, G$ and $e, f, g$ are

$$
\begin{array}{lll}
E=\overrightarrow{p_{\theta}} \cdot \overrightarrow{p_{\theta}} & F=\overrightarrow{p_{\theta}} \cdot \overrightarrow{p_{\varphi}} & G=\overrightarrow{p_{\varphi}} \cdot \overrightarrow{p_{\varphi}} \\
e=\vec{n} \cdot \overrightarrow{p_{\theta \theta}} & f=\vec{n} \cdot \overrightarrow{p_{\theta \varphi}} & g=\vec{n} \cdot \overrightarrow{p_{\varphi \varphi}},
\end{array}
$$

where

$$
\vec{n}=\frac{\overrightarrow{p_{\theta}} \times \overrightarrow{p_{\varphi}}}{\left|\overrightarrow{p_{\theta}} \times \overrightarrow{p_{\varphi}}\right|}
$$

After some rather large calculations we finally get: 


$$
\begin{aligned}
& E=\left(\frac{\partial R(\theta, \varphi)}{\partial \theta} \cos (\theta)-R(\theta, \varphi) \sin (\theta)\right)^{2}+ \\
&\left(\frac{\partial R(\theta, \varphi)}{\partial \theta} \sin (\theta) \cos (\varphi)+R(\theta, \varphi) \cos (\theta) \cos (\varphi)\right)^{2}+ \\
&\left(\frac{\partial R(\theta, \varphi)}{\partial \theta} \sin (\theta) \sin (\varphi)+R(\theta, \varphi) \cos (\theta) \sin (\varphi)\right)^{2}, \\
& F=\left(\frac{\partial R(\theta, \varphi)}{\partial \theta} \cos (\theta)-R(\theta, \varphi) \sin (\theta)\right) \frac{\partial R(\theta, \varphi)}{\partial \varphi} \cos (\theta)+ \\
&\left(\frac{\partial R(\theta, \varphi)}{\partial \theta} \sin (\theta) \cos (\varphi)+R(\theta, \varphi) \cos (\theta) \cos (\varphi)\right) \\
&\left(\frac{\partial R(\theta, \varphi)}{\partial \varphi} \sin (\theta) \cos (\varphi)-R(\theta, \varphi) \sin (\theta) \sin (\varphi)\right)+ \\
&\left(\frac{\partial R(\theta, \varphi)}{\partial \theta} \sin (\theta) \sin (\varphi)+R(\theta, \varphi) \cos (\theta) \sin (\varphi)\right) \\
&\left(\frac{\partial R(\theta, \varphi)}{\partial \varphi} \sin (\theta) \sin (\varphi)+R(\theta, \varphi) \sin (\theta) \cos (\varphi)\right) \\
& G=\left(\frac{\partial R(\theta, \varphi)}{\partial \varphi} \cos (\theta)\right)^{2}+\left(\frac{\partial R(\theta, \varphi)}{\partial \varphi} \sin (\theta) \cos (\varphi)-R(\theta, \varphi) \sin (\theta) \sin (\varphi)\right)^{2}+ \\
&\left(\frac{\partial R(\theta, \varphi)}{\partial \varphi} \sin (\theta) \sin (\varphi)+R(\theta, \varphi) \sin (\theta) \cos (\varphi)\right)^{2}
\end{aligned}
$$

Formulas for $e, f, g$ are omitted because they are very large (several pages). Finally we can compute the power and astigmatism as

$$
\begin{aligned}
\operatorname{Pow}(\theta, \varphi) & =(\mu-1) H(\theta, \varphi) \\
\operatorname{Ast}(\theta, \varphi) & =-2(\mu-1) \sqrt{H(\theta, \varphi)^{2}-K(\theta, \varphi)},
\end{aligned}
$$

where

$$
\begin{aligned}
& K(\theta, \varphi)=\operatorname{det}(A)=\frac{e g-f^{2}}{E G-F^{2}} \\
& H(\theta, \varphi)=\frac{1}{2} \operatorname{tr}(A)=\frac{e G-2 f F+g E}{2\left(E G-F^{2}\right)} .
\end{aligned}
$$

